\title{
Controlling Anderson localization in disordered photonic crystal waveguides
}

\author{
P. D. García* S. Smolka, S. Stobbe, and P. Lodah \\ DTU Fotonik, Department of Photonics Engineering, \\ Technical University of Denmark, Ørsteds Plads 343, DK 2800 Kgs. Lyngby, Denmark
}

(Dated: March 24, 2022)

\begin{abstract}
We prove Anderson localization in a disordered photonic crystal waveguide by measuring the ensemble-averaged localization length, $\xi$, which is controlled by the dispersion of the photonic crystal waveguide. In such structures, $\xi$ shows a 10 -fold variation between the fast- and the slow-light regime and, in the latter case, it becomes shorter than the sample length thus giving rise to strongly confined modes. The dispersive behavior of $\xi$ demonstrates the close relation between Anderson localization and the photon density of states in disordered photonic crystals, which opens a promising route to controlling and exploiting Anderson localization for efficient light confinement.
\end{abstract}

PACS numbers: (42.25.Dd, 42.25.Fx, 46.65.+g, 42.70.Qs)

Quantum optics and quantum information technologies require enhancement of light-matter interaction by, for example, confining light in a highly engineered nanocavity [1]. Quite remarkably, an alternative route towards light confinement exploits multiple scattering of light in disordered photonic structures, as originally proposed for electrons by P. W. Anderson [2]. The mechanism responsible for Anderson localization is wave interference and hence occurs not only for electrons but also for, e.g., microwaves [3], acoustic waves [4], and even Bose-Einstein condensated matter waves [5] thus illuminating the multidisciplinarity of the research field. In the case of light, indications of three-dimensional (3D) Anderson localization have been observed in random dielectric materials like powders composed of particles with casual shapes and sizes [6]. In these systems no control can be exerted over the frequency or spatial extent of the localized modes.

A promising proposal on how to control multiple scattering is to introduce disorder in photonic crystals [7]: the interference of multiply scattered light is expected to form Anderson-localized modes that appear randomly in space but at frequencies in or near the photonic crystal band gap. The characteristic length scale of Anderson localization is the localization length $\xi$, which is the exponential decay length of the confined modes after averaging over many realizations of disorder. In one- and two-dimensional (1D, 2D) systems, localization occurs for any degree of disorder when the sample length exceeds $\xi$ [8], in which case the photonic conductor becomes an insulator. Contrary to non-dispersive systems, photonic crystals offer the possibility to alter the photon density of states (DOS) allowing to control macroscopic transport properties [9] or spontaneous emission of photons [10], and in particular to achieve dispersive Anderson localization.

In this Letter, we show how to control and accurately tune Anderson localization of light to a spectral range of particular interest using dispersion in photonic crystal. In particular, we use a 1D disordered photonic crys- tal waveguide $(\mathrm{PCW})$ to prove the close relation between Anderson localization of light and the DOS. Indeed, photonic crystals owe their success to the fact that their DOS can be accurately tailored a priori and we show how this property can be exploited to tailor Anderson localization. By explicitly measuring the dispersive localization length, we unambiguously demonstrate that the strongly confined modes appearing in the slow-light regime of PCWs [1] are due to 1D Anderson localization, which has been questioned recently [12]. In addition, we interpret our experimental data on the wavelength-dependent localization length with a model for the DOS of a PCW thereby explicitly linking the localization length and the DOS.

Our samples consist of a membrane with a high refractive index material (GaAs, $n=3.54$ ) in which light is confined by total internal reflection. An ordered lattice of holes is etched in the structure forming a 2D photonic band gap that suppresses in-plane propagation of light. A waveguide is engineered in the structure by leaving out a row of holes. Light propagation in an ideal PCW is described by Bloch modes with a dispersion relation $\omega(k)$, where $\omega$ is the wave frequency and $k$ is the wave vector. PCWs can generate slow light, i.e., light with a very low group velocity, $v_{\mathrm{g}}=\partial \omega / \partial k=c / n_{\mathrm{g}}$, where $\mathrm{c}$ is the speed of light in vacuum. The slow-down factor $n_{\mathrm{g}}$, also referred to as the group index, is directly proportional to the DOS per unit length of the propagating mode in the PCW: DOS $=(1 / \pi) \partial k / \partial \omega=n_{\mathrm{g}} /(\pi c)$. Imperfections in a PCW lead to multiple scattering of light and the dispersion relation breaks down [13] thus inducing localized modes in the slow-light regime where the sample length exceeds $\xi$.

To investigate the impact of disorder on the light propagation in PCW we randomly vary the hole positions in the three rows above and below the waveguide using a Gaussian random number generator function (BoxMuller). The degree of disorder in each sample, $\delta$, is characterized by the standard deviation of the hole position with respect to the lattice constant varying from 


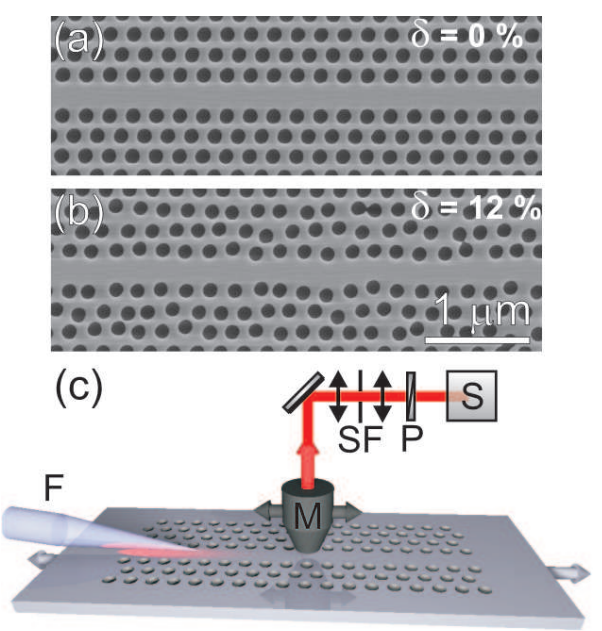

(d)

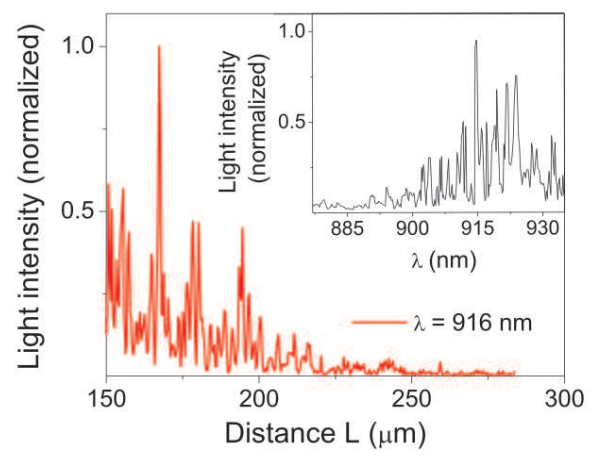

FIG. 1: (Color online) (a) and (b) Scanning electron microscope images of photonic crystal waveguides (top-view) with engineered disorder on the positions of the holes of $\delta=0 \%$ and $\delta=12 \%$ (standard deviation relative to the lattice constant). (c) Setup used to measure the localization length. The tip of a single mode tapered fiber $(\mathrm{F})$ is placed on the photonic crystal waveguide. TE-polarized light scattered from the waveguide is collected with an optical microscope objective (M), a spatial filter (SF), and a polarizer $(\mathrm{P})$ and sent to a spectrometer (S). (d) Measurement of strongly fluctuating light intensity in the slow-light regime of a PCW with $\delta=0 \%$ versus the distance from the light source. The inset of the figure shows a spectrum taken at $L=150 \mu \mathrm{m}$ from the fiber tip.

$\delta=0 \%$ to $\delta=12 \%$ (Fig. 1(a), (b)). The samples consist of a triangular lattice of holes with a lattice constant $a=240 \mathrm{~nm}$, a filling fraction $f=0.330 \pm 0.006$, membrane height $h=160 \pm 5 \mathrm{~nm}$, and a total length $L_{0}=1 \mathrm{~mm}$.

To characterize Anderson localization in PCWs we use the optical setup illustrated in Fig. 1(c). A continuous wave Ti:sapphire laser tuneable within $\lambda=700-1000 \mathrm{~nm}$ is coupled into a single mode tapered fiber with a tip diameter comparable to the waveguide width. The fiber evanescent mode couples to the waveguide mode by placing the fiber tip in close proximity of the PCW. We measure light scattered out-of-plane from the $\mathrm{PCW}$ with a
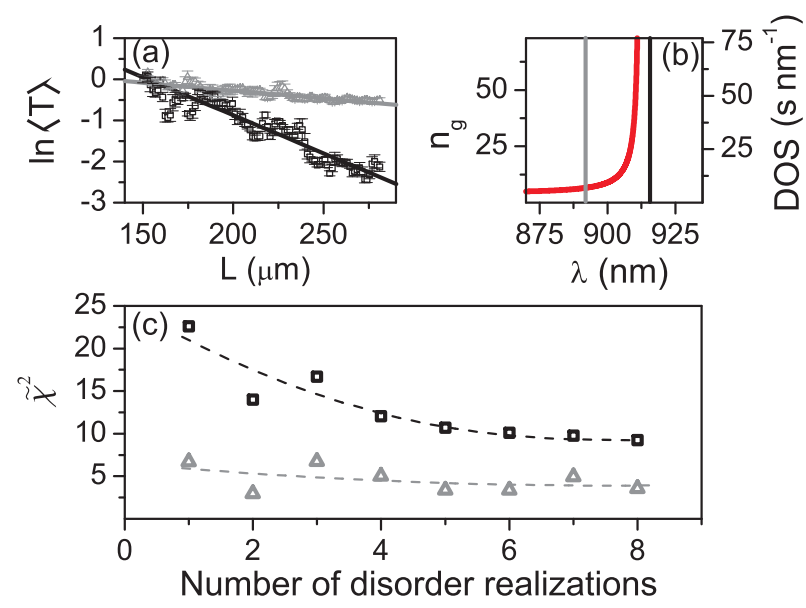

FIG. 2: (Color online) (a) Exponential fit of the ensembleaveraged light transmission in PCWs with $\delta=0 \%$ as a function of the distance from the source for two wavelengths: $\lambda_{1}=890 \mathrm{~nm}$ (gray points) and $\lambda_{2}=916 \mathrm{~nm}$ (black points). The spectral resolution is $2 \mathrm{~nm}$ and the slope equals the inverse of the localization length. (b) The calculated density of states (DOS) and group index $\left(n_{\mathrm{g}}\right)$ of an ideal structure without disorder. The two specific wavelengths $\lambda_{1}$ (gray line) and $\lambda_{2}$ (black line) are indicated that lie in the fast- and slow-light regimes, respectively. The calculated spectral position of the cutoff of the ideal waveguide mode gives the best fit to our experimental data (explained in the text). (c) Plot of the reduced chi-square, $\widetilde{\chi}^{2}$, for the exponential fit to the ensemble-averaged light transmission in the fast- (gray triangles) and slow-light regime (black squares) versus number of realizations of disorder. The dashed lines are guides to the eyes.

high amplification microscope objective $(\mathrm{NA}=0.8)$ as a function of the wavelength and the distance $L$ from the light source, i.e., the fiber tip. The measurement starts at $L=150 \mu \mathrm{m}$ from the fiber tip to avoid any spurious effect due to the evanescent field or light that is not coupled to the waveguide mode. Fig. 1(d) shows a measurement of scattered light intensity versus $L$ in the slow-light regime (at $\lambda=916 \mathrm{~nm}$ where $n_{\mathrm{g}}>15$ ) for a single realization of disorder. In this case, $\delta=0 \%$, the PCW is only affected by intrinsic unavoidable disorder introduced in the fabrication process. The inset of the figure shows a spectrum of the scattered light intensity at $L=150 \mu \mathrm{m}$. The strong fluctuation in the light intensity is a signature of 1D Anderson-localized modes. The modes appear to be spatially and spectrally separated, which constitutes a criterion for Anderson localization [14].

In the Anderson localization regime the fluctuating light transmission, $T(L)$, decays exponentially after ensemble-averaging, giving $\ln \langle T\rangle=-L / 2 \xi[15]$. We have used this property to extract the localization length and confirm the Anderson localization criterion of $\xi<L$. To do this, we perform ensemble averaging by measuring the light leakage versus $L$ and wavelength for 8 different re- 


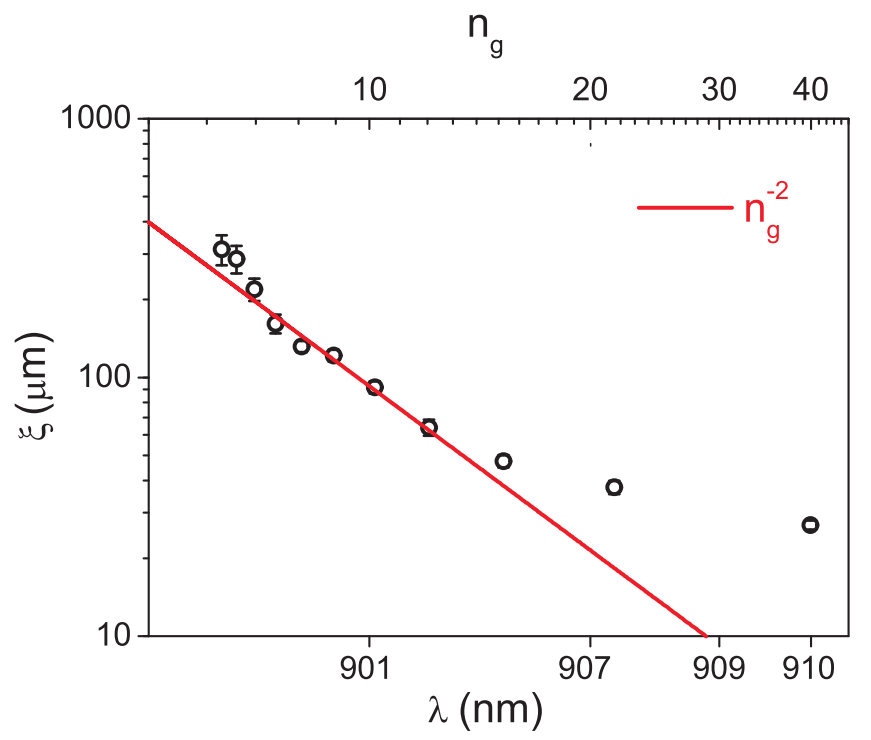

FIG. 3: (Color online) Plot of the localization length, $\xi$, of the PCW with $\delta=0 \%$ as a function of the wavelength and the group index of the ideal structure $n_{\mathrm{g}}$. A strongly dispersive localization length is found that reaches a minimum in the slow-light regime $\left(n_{\mathrm{g}}>15\right)$. The scaling $\xi \propto n_{\mathrm{g}}^{-2}$ (red curve) is obtained from a model of the light scattering cross section in the $\mathrm{PCW}$.

alizations of disorder. In particular, we probe different spatial realizations of disorder by moving the fiber together with the microscope objective along the waveguide and repeating the measurement. Fig. 2(a) shows the linear fit of $\ln \langle T\rangle$ in the sample with $\delta=0 \%$ in the fast-light regime $n_{\mathrm{g}}\left(\lambda_{1}\right)<15$ and slow-light regime $n_{\mathrm{g}}\left(\lambda_{2}\right)>15$ (corresponding to low and high DOS, respectively, see Fig. 2(b)). For these spectral positions we extract the localization lengths $\xi\left(\lambda_{1}\right)=(263 \pm 28) \mu \mathrm{m}$ and $\xi\left(\lambda_{2}\right)=(27 \pm 2) \mu \mathrm{m}$. We obtain $n_{\mathrm{g}}$ from numerical simulations of the disorder-free ideal structure, which quantifies the spectral position with respect to the cutoff of the ideal waveguide mode. This spectral position is obtained from the best fit to our experimental data with a 1D model (explained later on). It is within the uncertainty in the waveguide mode band edge position, which is caused by uncertainties in the fabrication parameters given previously.

Fig. 2(c) shows the goodness of the fits, $\widetilde{\chi}^{2}$, performed in Fig. 2(a) to test the degree of convergence of the ensemble average to a single-exponential decay. The fluctuations in the data are due to speckles not fully ensembleaveraged. A disorder realization in our experiment consists of a scan of $T(L)$ varying $L$ over $130 \mu \mathrm{m}$. Thus, the number of different disorder realizations that we can perform without adding repeated statistics to the ensemble average is limited by the sample length, $L_{0}=1 \mathrm{~mm}$. Furthermore, the goodness of the fit does not reach the optimum value $\widetilde{\chi}^{2}=1$ corresponding to a single-exponential decay, which may be due to different reasons. Deviations from perfect verticality of the air holes can break the symmetry in the out-of-plane direction and couple TE- to TM-polarized waveguide modes giving rise to strongly localized modes 16. This polarization mixing mechanism may lead to multi-exponential decay of the ensembleaveraged transmission that is not resolved in the present experiment.

Fig. 3 contains the main contribution of this Letter: it shows the dispersive behavior of the measured localization length. We observe a 10-fold variation in the localization length with wavelength. In the slow-light regime, $\xi$ reaches its minimum value of $\sim(27 \pm 2) \mu \mathrm{m}$ and becomes much smaller than $L_{0}$, giving rise to strongly Andersonlocalized modes. The dispersive behavior of the localization length gives directly control over the extension of the modes, which can be precisely tuned by varying the wavelength. We can also tune the waveguide mode by varying the fabrication parameters (typically $a$ and $f$ ) thus, controlling the frequency of localized modes.

Any loss mechanisms of the light trapped in the PCW will in general influence the measured localization length depending on the spatial length scale of the loss process. In presence of light losses, we have $\ln \langle T\rangle=-L / 2 \ell$ where the decay length $\ell$ is defined as $\ell^{-1}=\xi^{-1}+\ell_{i}^{-1}+\ell_{e}{ }^{-1}$, where $\ell_{i}$ is the material inelastic absorption length and $\ell_{e}$ is the extinction length associated with out-of-plane losses. GaAs has a very low optical absorption coefficient $\left(<100 \mathrm{~cm}^{-1}\right.$ at a wavelength of $\left.\lambda=915 \mathrm{~nm}\right)$ corresponding to $\ell_{i}>1 \mathrm{~m}$. This value might be reduced by surface effects at the holes of the photonic crystal but is still expected to be much larger than $L_{0}$. Furthermore, we quantify $\ell_{e}$ applying our fabrication parameters to recent 3D numerical simulations of Bloch-mode scattering in PCWs 17]. In particular, for $\delta=0 \%$, for which the standard deviation of the hole positions is smaller than $2 \mathrm{~nm}$, we obtain $\ell_{e} \sim 400 \mathrm{~mm}$. Both $\ell_{i}$ and $\ell_{e}$ are much larger than $L_{0}$, thus not affecting the localization length we extract from our data, i.e., we can approximate $\ell \cong \xi$ at least for weak disorder.

In the following, we model the function $\xi\left(n_{\mathrm{g}}\right)$. The relation $\xi \approx N \cdot \ell_{\mathrm{s}}$ holds for $1 \mathrm{D}$ systems [15, 18], where $N$ is the number of electromagnetic modes that the system can sustain and $\ell_{\mathrm{S}}$ is the scattering mean free path. Our samples are single-mode PCWs and hence the localization length is $\xi \approx \ell_{\mathrm{s}}$. The dispersive behavior of $\ell_{\mathrm{s}}$ in $3 \mathrm{D}$ photonic crystals has been measured and explained in terms of the DOS very recently [19]. Two separate mechanisms determine $\ell_{\mathrm{s}}$ : the excitation of the scatterer and the subsequent radiation from the scatterer [20]. The coupling to the scatterer is described by the DOS of the mode of the excitation beam 21], which in our system is proportional to the group index of the waveguide mode. The second process is described by the local density of states (LDOS). Ignoring the minor contributions of coupling to unconfined radiation modes, the LDOS is also 
determined by the group index of the waveguide 22, 23]. This applies to every scattering event giving rise to a modified scattering cross section $\sigma$ in PCWs scaling as $\sigma \propto n_{\mathrm{g}}{ }^{2}(\omega)$. The scattering mean free path in a random medium can be expressed as $\ell_{\mathrm{s}}=1 / \rho_{\mathrm{s}} \sigma$ [24], where $\rho_{\mathrm{s}}$ is the density of scatterers. For $1 \mathrm{D}$ single-mode PCWs we therefore predict $\xi \propto n_{g}{ }^{-2}(\omega)$, which is in very good agreement with our experimental data in the fast-light regime. The red curve in Fig. 3 represents the best fit to our experimental results. It gives the spectral position of the waveguide mode cutoff used in Fig. 2(b). The fitting parameters are the number of data fitted and the spectral position of the cutoff of the waveguide mode. The same scaling of $\xi \propto n_{g}{ }^{-2}$ can also be recovered from $1 \mathrm{D}$ random matrix theory [25] and it is confirmed by 3D numerical simulations in PCWs 17]. Our model explains not only the dispersion in the localization length but also the scaling of light losses with group index in PCWs as $v_{\mathrm{g}}{ }^{-2}\left(n_{\mathrm{g}}{ }^{2}\right)$. Such a scaling has been systematically observed in the literature [26] and our model provides a physical explanation. From the data in Fig. 3 we observe that the model breaks down deep in the slow-light regime where strongly localized Anderson modes appear. This is expected since the model is based on the calculated DOS of the ideal structure without disorder, which is modified in the regime of strong multiple scattering and Anderson localization.

Finally, Fig. 4 plots the measurement of the localization length in samples with increasing amount of disorder and the mean value of the localization length, $\bar{\xi}$. The latter decreases with disorder, reaches a minimum for $\delta=6 \%$, and increases for $\delta>6 \%$. This behavior is a clear proof that losses are not dominant in our experiment even for strong disorder where $\ell_{e}$ is predicted to shorten [17]. The increase of $\bar{\xi}$ for $\delta>6 \%$ cannot be explained by an increase of light losses (decrease of $\ell_{e}$ ). That would lead to a decrease of the measured decay length as opposed to our observations. The behavior of $\bar{\xi}$ for weak disorder is predicted in Ref. [17] and we propose here a possible explanation for the increase observed for large amounts of disorder. Thus, the local disorder introduced in our samples only in the three rows above and below the waveguide could imply that several spatial modes, $N$, are effectively introduced when increasing disorder. This would increase the localization length according to $\bar{\xi} \propto N \cdot \ell_{\mathrm{S}}$ effectively decoupling $\xi$ and $\ell_{\mathrm{S}}$ and therefore enable a regime of light diffusion where $\ell_{\mathrm{s}}<L<\xi$. This could open a new route to investigate the crossover between diffusion and localization regimes in a dispersive quasi-1D system, which is not possible in standard quasi-1D disordered systems [18, 27].

In conclusion, we have demonstrated the close relation between Anderson localization of light and the electromagnetic DOS in PCWs. We explain multiple light scattering in PCWs with a DOS dependent scattering cross section explaining the strong dispersion of the lo-

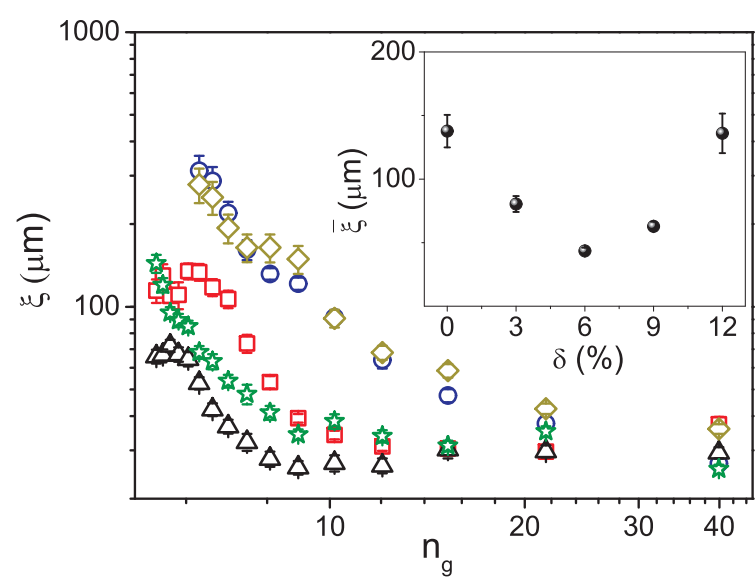

FIG. 4: (Color online) Localization length, $\xi$, as a function of the group index, $n_{\mathrm{g}}$, measured in PCWs with various amounts of disorder $\delta=0 \%(\circ), 3 \%(\square), 6 \%(\triangle), 9 \%(\star)$, and $12 \%$

$(\diamond)$. The inset shows the mean value of $\xi$ as a function of $\delta$.

calization length and the appearance of strongly confined modes in the slow-light regime. These results are of fundamental importance since they impose limitations for slow-light devices based on PCWs such as singlephoton sources [23]. At the contrary the strongly confined Anderson-localized cavities with tailored properties appear very appealing candidates for experiments on cavity quantum electrodynamics [28] or random lasing.

We thank Johan Raunkjær Ott for fruitful discussions. We gratefully acknowledge the Council for Independent Research (Technology and Production Sciences and Natural Sciences) for financial support.

* Electronic address: dgar@fotonik.dtu.dk

$\dagger$ Electronic address: pelo@fotonik.dtu.dk URL: http://www.fotonik.dtu.dk/quantumphotonics

[1] Y. Akahane, T. Asano, B. Song, and S. Noda, Nature 425, 944 (2003).

[2] P.W. Anderson, Phys. Rev. 109, 1492 (1958).

[3] A. A. Chabanov, M. Stoytchev, and A. Z. Genack, Nature 404, 850 (2000).

[4] H. Hu et al. Nature Physics 4, 945 (2008).

[5] J. Billy et al. Nature 453, 891 (2008); G. Roati et al. Nature 453, 895 (2008).

[6] D. S. Wiersma et al. Nature, 390, 671 (1997); M. Storzer, P. Gross, C. M. Aegerter, and G. Maret, Phys. Rev. Lett. 96, 063904 (2006).

[7] S. John, Phys. Rev. Lett. 58, 2486 (1987).

[8] N. F. Mott and W. D. Twose, Avd. Phys. 10, 107 (1961).

[9] A. Lagendijk and B.A. van Tiggelen, Phys. Rep. 270, 143 (1996); R. Carminati and J. J. Saenz, Phys. Rev. Lett. 102, 093902 (2009).

[10] P. Lodahl et. al. Nature, 430, 654 (2004).

[11] J. Topolancik, B. Ilic, and F. Vollmer, Phys. Rev. Lett. 99, 253901 (2007). 
[12] M. Patterson et al. Phys. Rev. Lett. 102, 253903 (2009).

[13] N. Le Thomas et al. Phys. Rev. B 80, 125332 (2009).

[14] D. J. Thouless, Phys. Rev. Lett. 39, 1167 (1977).

[15] C. W. J. Beenakker, Rev. Mod. Phys. 69, 731 (1997).

[16] J. Topolancik, F. Vollmer, R. Ilic, and M. Crescimanno, Opt. Express 17, 12470 (2009)

[17] S. Mazoyer, J. P. Hugonin, and P. Lalanne, Phys. Rev. Lett. 103, 063903 (2009).

[18] A. Garcia-Martin and J. J. Saenz, Phys. Rev. Lett. 87, 116603 (2001)

[19] P. D. Garcia, R. Sapienza, L. S. Froufe-Perez, and C. Lopez, Phys. Rev. B 79, 241109(R) (2009).

[20] L. S. Froufe-Perez, R. Sapienza, P. D. Garcia, and C. Lopez, (Unpublished).

[21] R. C. McPhedran et al. Phys. Rev. E 69, 016609 (2004).

[22] V. S. C. Manga Rao and S. Hughes, Phys. Rev. B 75, 205437 (2007)
[23] T. Lund-Hansen et al. Phys. Rev. Lett. 101, 113903 (2008).

[24] P. Sheng, Introduction to Wave Scattering, Localization, and Mesoscopic Phenomena Academic Press, San Diego (1995).

[25] L. S. Froufe-Perez, M. Yepez, P. A. Mello, and J. J. Saenz, Phys. Rev. E 75, 031113 (2007).

[26] E. Kuramochi et al. Phys. Rev. B 72, 161318(R) (2005); R. J. P. Engelen, D. Mori, T. Baba, and L. Kuipers, Phys. Rev. Lett. 101, 103901 (2008); L. O'Faolain et al. Opt. Express 15, 13129 (2007).

[27] J. Bertolotti, S. Gottardo, D. S. Wiersma, M. Ghulinyan, and L. Pavesi, Phys. Rev. Lett. 94, 113903 (2005).

[28] L. Sapienza, H. Thyrrestrup, S. Stobbe, P. D. Garcia, S. Smolka, and P. Lodahl, Science 327, 1352 (2010). 